\title{
INF-SUP CONDITION FOR SPHERICAL POLYNOMIALS AND RADIAL BASIS FUNCTIONS ON SPHERES
}

\author{
IAN H. SLOAN AND HOLGER WENDLAND
}

\begin{abstract}
Interpolation by radial basis functions and interpolation by polynomials are both popular methods for function reconstruction from discrete data given on spheres. Recently, there has been an increasing interest in employing these function families together in hybrid schemes for scattered data modeling and the solution of partial differential equations on spheres. For the theoretical analysis of numerical methods for the associated discretized systems, a so-called inf-sup condition is crucial. In this paper, we derive such an inf-sup condition, and show that the constant in the inf-sup condition is independent of the polynomial degree and of the chosen point set, provided the mesh norm of the point set is sufficiently small. We then use the inf-sup condition to derive a new error analysis for the hybrid interpolation scheme of Sloan and Sommariva.
\end{abstract}

\section{InTRODUCTION}

The inf-sup condition is a fundamental tool for the stability and convergence analysis of mixed finite element methods for PDEs 1, 2, 4. In this paper we prove an inf-sup condition in a completely different context, that of approximation by spherical polynomials and radial basis functions on spheres of arbitrary dimension, as discussed in [11].

The inf-sup condition is presented in the next section. In the following paragraphs we introduce just enough notation and definitions to make the result understandable.

We will work on the unit sphere $S^{d}:=\left\{x \in \mathbb{R}^{d+1}:\|x\|_{2}=1\right\}$ and use the usual function spaces $C\left(S^{d}\right), L_{2}\left(S^{d}\right)$.

The set of $(d+1)$-variate polynomials of degree less than or equal to $L \in \mathbb{N}_{0}$, restricted to the sphere $S^{d}$, will be denoted by $\mathbb{P}_{L}$. This space can be equipped with an $L_{2}\left(S^{d}\right)$-orthonormal basis $\left\{Y_{\ell, k}: 0 \leq \ell \leq L, 1 \leq k \leq N(d, \ell)\right\}$, consisting of the so-called spherical harmonics. Here, $\ell$ refers to the degree, while $k$ indicates a numeration of the orthonormal basis functions of the same degree, which span a linear space of dimension $N(d, \ell)$. It is well known (see [9]) that the collection of all spherical harmonics forms a complete orthonormal basis of $L_{2}\left(S^{d}\right)$, hence every

Received by the editor December 17, 2007 and, in revised form, August 6, 2008.

2000 Mathematics Subject Classification. Primary 65D05, Secondary 41A05, 41A29.

Key words and phrases. Inf-sup, sphere, radial basis function, spherical polynomial, hybrid approximation. 
function $u \in L_{2}\left(S^{d}\right)$ can be represented by its Fourier series

$$
u=\sum_{\ell=0}^{\infty} \sum_{k=1}^{N(d, \ell)} \widehat{u}_{\ell, k} Y_{\ell, k}, \quad \widehat{u}_{\ell, k}=\int_{S^{d}} u(x) Y_{\ell, k}(x) d x,
$$

where $d x$ denotes the area measure on $S^{d}$.

This representation can be used to introduce Sobolev spaces on $S^{d}$. To be more precise, we define the Sobolev space $H^{s}$ for $s \geq 0$ by

$$
H^{s}:=\left\{u \in L_{2}\left(S^{d}\right): \sum_{\ell=0}^{\infty} \sum_{k=1}^{N(d, \ell)}\left(1+\ell^{2}\right)^{s}\left|\widehat{u}_{\ell, k}\right|^{2}<\infty\right\} .
$$

Obviously, this space is equipped with a norm stemming from the inner product

$$
(u, v)_{H^{s}}:=\sum_{\ell=0}^{\infty} \sum_{k=1}^{N(d, \ell)}\left(1+\ell^{2}\right)^{s} \widehat{u}_{\ell, k} \widehat{v}_{\ell, k} .
$$

Putting $s=0$ we obtain $H^{0}=L_{2}\left(S^{d}\right)$.

It is also known that if $s>d / 2$, the Sobolev embedding theorem gives $H^{s} \subset$ $C\left(S^{d}\right)$. In this case $H^{s}$ possesses a reproducing kernel, i.e. a symmetric function $\Phi=\Phi_{s}: S^{d} \times S^{d} \rightarrow \mathbb{R}$ with the properties

$$
\Phi(\cdot, x) \in H^{s} \quad \text { for } x \in S^{d}, \quad u(x)=(u, \Phi(\cdot, x))_{H^{s}} \quad \text { for } u \in H^{s}, x \in S^{d} .
$$

It is easy to verify that this kernel has the Fourier representation

$$
\Phi(x, y)=\sum_{\ell=0}^{\infty} \sum_{k=1}^{N(d, \ell)} \frac{1}{\left(1+\ell^{2}\right)^{s}} Y_{\ell, k}(x) Y_{\ell, k}(y) .
$$

This kernel is (strictly) positive definite, in the sense that for all possible choices of distinct points $X=\left\{x_{1}, \ldots, x_{N}\right\} \subset S^{d}$ the $N \times N$ matrices of the form $\left(\Phi\left(x_{i}, x_{j}\right)\right)$ are positive definite. Consequently, for a given fixed set $X$ and given data values $f_{1}, \ldots, f_{N}$ at $x_{1}, \ldots, x_{N}$, there always exists a unique interpolant from the space

$$
V_{X}:=\operatorname{span}\left\{\Phi\left(\cdot, x_{1}\right), \ldots, \Phi\left(\cdot, x_{N}\right)\right\} .
$$

The approximation error for such an interpolant is in general expressed in terms of the fill distance

$$
h_{X}:=\sup _{x \in S^{d}} \min _{x_{j} \in X} \operatorname{dist}\left(x, x_{j}\right),
$$

where $\operatorname{dist}(x, y)$ denotes the geodesic distance $\cos ^{-1}(x \cdot y)$ between two points $x, y$ on the sphere.

We have introduced two finite-dimensional function spaces: the spherical polynomials $\mathbb{P}_{L}$ of degree at most $L$, and the kernel-based space $V_{X}$. If both of these spaces are employed in a mixed approximation scheme, an inf-sup condition is necessary to make significant statements on the convergence of numerical schemes. We will establish such a condition and use it to obtain an error analysis of a hybrid approximation scheme.

We remark that a weaker inf-sup condition is relatively easy to establish, via the "sampling theorem"; see [8, 12. The result is weaker in the sense that the constant in the inf-sup condition now depends on the polynomial degree $L$. Nonetheless, for completeness, we state the sampling theorem and derive this weaker result in Section 4. 
In Section 5 we use the inf-sup condition to obtain a new error analysis, this time for the $L_{2}$ norm of the error, for the hybrid interpolation scheme of Sloan and Sommariva [1]. Finally, in Section [6 the results of the paper are extended to approximation with more general kernels $\Phi(\mathbf{x}, \mathbf{y})$ for which the norm in the associated "native space" is merely equivalent (rather then identical) to the $H^{s}$ norm.

\section{MAIN RESULTS}

In this section, we will state the inf-sup condition for interpolation by radial basis functions and spherical polynomials in three equivalent forms.

Theorem 2.1. There exist constants $\gamma>0$ and $\tau>0$ depending only on $d$ and $s>d / 2$ and such that for all $L \geq 1$ and all $X=\left\{x_{1}, \ldots, x_{N}\right\} \subset S^{d}$ satisfying $h_{X} \leq \tau / L$ the following inequality holds:

$$
\sup _{v \in V_{X} \backslash\{0\}} \frac{(p, v)_{H^{s}}}{\|v\|_{H^{s}}} \geq \gamma\|p\|_{H^{s}}, \quad p \in \mathbb{P}_{L} .
$$

Consequently, taking the infimum over all $p \in \mathbb{P}_{L}$, we can replace (4) by

$$
\inf _{p \in \mathbb{P}_{L} \backslash\{0\}} \sup _{v \in V_{X} \backslash\{0\}} \frac{(p, v)_{H^{s}}}{\|v\|_{H^{s}}\|p\|_{H^{s}}} \geq \gamma .
$$

Later in this section we shall prove that this theorem can equivalently be stated as in either of the next two theorems.

Theorem 2.2. There exist constants $\gamma>0$ and $\tau>0$ depending only on $d$ and $s$ such that for $s>d / 2$ and all $L \geq 1$ and all $X=\left\{x_{1}, \ldots, x_{N}\right\} \subset S^{d}$ satisfying $h_{X} \leq \tau / L$ the following inequality holds:

$$
\|P\|_{H^{s}} \geq \gamma\|p\|_{H^{s}}
$$

for all $p \in \mathbb{P}_{L}$ and all $P \in H^{s}$ satisfying $P|X=p| X$.

As pointed out earlier, for every continuous function $f \in C\left(S^{d}\right)$, there exists a unique interpolant $I_{X} f$ of the form

$$
I_{X} f=\sum_{j=1}^{N} \alpha_{j} \Phi\left(\cdot, x_{j}\right),
$$

where the coefficients $\alpha_{j}$ are determined by the interpolation conditions $I_{X} f \mid X=$ $f \mid X$. It is well known that the interpolant $I_{X} f$ from $V_{X}$ to $f \in C\left(S^{d}\right)$ is also the norm-minimal interpolant (see, for example, [12, Theorem 13.2]), i.e., it satisfies

$$
\left\|I_{X} f\right\|_{H^{s}}=\min \left\{\|P\|_{H^{s}}: P \in H^{s} \text { and } P|X=f| X\right\}, \quad f \in C\left(S^{d}\right) .
$$

From this minimal norm property it immediately follows that Theorem 2.2 can equivalently be reformulated as

Theorem 2.3. There exist constants $\gamma>0$ and $\tau>0$ depending only on $d$ and $s$ such that for $s>d / 2$ and all $L \geq 1$ and all $X=\left\{x_{1}, \ldots, x_{N}\right\} \subset S^{d}$ satisfying $h_{X} \leq \tau / L$ the following inequality holds:

$$
\left\|I_{X} p\right\|_{H^{s}} \geq \gamma\|p\|_{H^{s}}
$$

for all $p \in \mathbb{P}_{L}$. 
We will use the following rather simple Hilbert space proposition to establish the equivalence of Theorems 2.1 and 2.3 Since we already know that Theorems 2.2 and 2.3 are equivalent, this will establish the equivalence of all three theorems.

Proposition 2.4. Let $\mathbb{X}$ and $\mathbb{Y}$ be subspaces of a Hilbert space $\mathbb{H}$ with inner product $(\cdot, \cdot)$ and norm $\|\cdot\|=(\cdot, \cdot)^{1 / 2}$. Then, for all $y \in \mathbb{Y}$,

$$
\sup _{x \in \mathbb{X} \backslash\{0\}} \frac{(y, x)}{\|x\|}=\|Y\|,
$$

where $Y$ is the orthogonal projection of $y$ on $\mathbb{X}$, i.e.,

$$
Y \in \mathbb{X} \quad \text { and } \quad(Y-y, x)=0 \quad \text { for all } x \in \mathbb{X} .
$$

Proof. From (77) and the Cauchy-Schwarz inequality, for $0 \neq x \in \mathbb{X}$ and fixed $y \in \mathbb{Y}$ we have

$$
\frac{(y, x)}{\|x\|}=\frac{(Y, x)}{\|x\|} \leq\|Y\| \text {. }
$$

Thus,

$$
\sup _{x \in \mathbb{X} \backslash\{0\}} \frac{(y, x)}{\|x\|} \leq\|Y\|,
$$

in which the inequality can be replaced by equality, since equality is achieved by $x=Y$, using $(y, Y)=(Y, Y)=\|Y\|^{2}$.

To apply this result to our situation, we take $\mathbb{H}=H^{s}, \mathbb{X}=V_{X}, \mathbb{Y}=\mathbb{P}_{L}$. Since $\mathbb{H}$ is a reproducing kernel space with reproducing kernel $\Phi$, we see that

$$
\left(I_{X} f-f, \Phi\left(\cdot, x_{j}\right)\right)_{H^{s}}=I_{X} f\left(x_{j}\right)-f\left(x_{j}\right)=0, \quad x_{j} \in X,
$$

since $I_{X} f$ interpolates $f$. This implies, given (3), that

$$
\left(I_{X} f-f, v\right)_{H^{s}}=0 \quad \text { for all } v \in V_{X} .
$$

Thus, $I_{X} f$ is the orthogonal projection of $f$ on $V_{X}$ in $H^{s}$, or equally, the best approximation to $f$ from $V_{X}$ in the $H^{s}$ norm. Proposition 2.4 therefore gives

$$
\sup _{v \in V_{X} \backslash\{0\}} \frac{(p, v)_{H^{s}}}{\|v\|_{H^{s}}}=\left\|I_{X} p\right\|_{H^{s}}, \quad p \in \mathbb{P}_{L} .
$$

The equivalence of Theorem 2.3 and Theorem 2.1 is now obvious.

\section{Proof of The Inf-Sup TheOrem}

We will use the following $L_{2}\left(S^{d}\right)$ estimate from [5, 7].

Theorem 3.1. There exist constants $C>0$ and $h_{0}>0$ depending only on $s$ and $d$ such that for $s>d / 2$,

$$
\|g\|_{L_{2}} \leq C h_{X}^{s}\|g\|_{H^{s}}
$$

for all $X \subset S^{d}$ with $h_{X} \leq h_{0}$, and all $g \in H^{s}$ satisfying $g \mid X=0$.

The proof of this theorem rests on a corresponding result [10] for Euclidean spaces, which in turn relies mainly on two steps. In the first step an error estimate of this form for the $L_{\infty}$-norm is established, with an appropriately reduced exponent of $h_{X}$. This can be done using either the so-called power function approach, or using the sampling theorem, which we will discuss in more detail in the next section. The second step consists of a local covering argument due to Duchon (see [3, 6]), which 
then provides the additional convergence order reflecting the fact that the left-hand side is measured in the weaker $L_{2}$-norm.

Theorem 3.1 leads to the following corollary.

Corollary 3.2. Suppose $f \in H^{s}$ with $s>d / 2$. Then we have, for all $X=$ $\left\{x_{1}, \ldots, x_{N}\right\} \subset S^{d}$ with $h_{X} \leq h_{0}$, the estimate

$$
\left\|f-I_{X} f\right\|_{L^{2}} \leq C h_{X}^{s}\left\|f-I_{X} f\right\|_{H^{s}} \leq C h_{X}^{s}\|f\|_{H^{s}},
$$

where $h_{0}$ and $C$ are the constants from Theorem 3.1 .

Note that in the last step in (9) we use the fact that $I_{X} f$ is the orthogonal projection in $H^{s}$, implying $\left\|f-I_{X} f\right\|_{H^{s}} \leq\|f\|_{H^{s}}$.

The $L_{2}$-estimate from Corollary 3.2 gives rise to an estimate in the $H^{s}$-norm for functions from $H^{2 s}$; see also [6]. This follows simply from (8), together with the Cauchy-Schwarz inequality:

$$
\begin{aligned}
\left\|f-I_{X} f\right\|_{H^{s}}^{2} & =\left(f-I_{X} f, f\right)_{H^{s}}=\sum_{\ell=0}^{\infty} \sum_{k=1}^{N(d, \ell)}\left(1+\ell^{2}\right)^{s}\left(f-I_{X} f\right)_{\ell, k} \widehat{f}_{\ell, k} \\
& \leq\left(\sum_{\ell=0}^{\infty} \sum_{k=1}^{N(d, \ell)}\left|\widehat{f}_{\ell, k}\right|^{2}\left(1+\ell^{2}\right)^{2 s}\right)^{1 / 2}\left(\sum_{\ell=0}^{\infty} \sum_{k=1}^{N(d, \ell)}\left|\left(f-I_{X} f\right)_{\ell, k}\right|^{2}\right)^{1 / 2} \\
& =\|f\|_{H^{2 s}}\left\|f-I_{X} f\right\|_{L_{2}} \\
& \leq C h_{X}^{s}\|f\|_{H^{2 s}}\left\|f-I_{X} f\right\|_{H^{s}},
\end{aligned}
$$

provided that $h_{X} \leq h_{0}$. The following result now follows from cancellation of $\left\|f-I_{X} f\right\|_{H^{s}}$.

Corollary 3.3. Suppose $f \in H^{2 s}$ with $s>d / 2$. Then we have, for all $X=$ $\left\{x_{1}, \ldots, x_{N}\right\} \subset S^{d}$ with $h_{X} \leq h_{0}$, the estimate

$$
\left\|f-I_{X} f\right\|_{H^{s}} \leq C h_{X}^{s}\|f\|_{H^{2 s}},
$$

where $h_{0}$ and $C$ are the constants from Theorem 3.1 .

Note that we can insert (10) into (9) to derive

$$
\left\|f-I_{X} f\right\|_{L_{2}} \leq C^{2} h_{X}^{2 s}\|f\|_{H^{2 s}},
$$

which means that we double the approximation order in the $L_{2}$-norm for target functions having twice the smoothness. This well-known $L_{2}$-estimate will not play a role in our subsequent arguments, but Corollary 3.3 will do so.

The second ingredient in our proof will be an inverse estimate for spherical polynomials: For every $p \in \mathbb{P}_{L}$ and every $t>s \geq 0$ we have

$$
\begin{aligned}
\|p\|_{H^{t}}^{2} & =\sum_{\ell=0}^{L} \sum_{k=1}^{N(d, \ell)}\left|\widehat{p}_{\ell, k}\right|^{2}\left(1+\ell^{2}\right)^{t} \\
\leq & \left(1+L^{2}\right)^{t-s} \sum_{\ell=0}^{L} \sum_{k=1}^{N(d, \ell)}\left|\widehat{p}_{\ell, k}\right|^{2}\left(1+\ell^{2}\right)^{s}=\left(1+L^{2}\right)^{t-s}\|p\|_{H^{s}}^{2} .
\end{aligned}
$$


Proof of Theorem 2.3. Since $\mathbb{P}_{L} \subset H^{2 s}$ for all $L \geq 1$, we can combine the error estimate (10) with the inverse estimate (11) with $t=2 \mathrm{~s}$ to give, for arbitrary $p \in \mathbb{P}_{L}$ and $h_{X} \leq h_{0}$,

$$
\left\|p-I_{X} p\right\|_{H^{s}} \leq C h_{X}^{s}\|p\|_{H^{2 s}} \leq C h_{X}^{s}(\sqrt{2} L)^{s}\|p\|_{H^{s}},
$$

where we used $1+L^{2} \leq 2 L^{2}$. Now we choose a fixed $\delta \in(0,1)$, and deduce

$$
\left\|p-I_{X} p\right\|_{H^{s}} \leq \delta\|p\|_{H^{s}}
$$

provided that

$$
h_{X} \leq \min \left\{h_{0},\left(\frac{\delta}{C}\right)^{1 / s} \frac{1}{\sqrt{2} L}\right\} .
$$

Because $I_{X} p$ is the orthogonal projection of $p$ in $H^{s}$, we can use the Pythagoras theorem

$$
\|p\|_{H^{s}}^{2}=\left\|p-I_{X} p\right\|_{H^{s}}^{2}+\left\|I_{X} p\right\|_{H^{s}}^{2}
$$

to obtain

$$
\left\|I_{X} p\right\|_{H^{s}}^{2}=\|p\|_{H^{s}}^{2}-\left\|p-I_{X} p\right\|_{H^{s}}^{2} \geq\left(1-\delta^{2}\right)\|p\|_{H^{s}}^{2} .
$$

Theorem 2.3 is therefore proved with

$$
\gamma=\sqrt{1-\delta^{2}}, \tau=\min \left\{h_{0}, \frac{1}{\sqrt{2}}\left(\frac{\delta}{C}\right)^{1 / s}\right\} .
$$

In the proof above, $\delta$ is an arbitrary number in $(0,1)$, hence so too is $\gamma$. For applications we may wish to be more careful in the choice of parameters. Suppose $\gamma_{0}$ is the minimum value of $\gamma$ we would wish to allow in Theorem 2.3 for some application; for example, perhaps $\gamma_{0}=0.1$. Then $\delta_{0}:=\sqrt{1-\gamma_{0}^{2}}$ is the maximum permissible value of $\delta$. If $h_{0} \geq(1 / \sqrt{2})\left(\delta_{0} / C\right)^{1 / s}$, then a natural choice is $\delta=\delta_{0}$, in which case $\gamma=\gamma_{0}$ and $\tau=(1 / \sqrt{2})\left(\delta_{0} / C\right)^{1 / s}<h_{0}$. If, on the other hand, $h_{0}<(1 / \sqrt{2})\left(\delta_{0} / C\right)^{1 / s}$, then we may without loss take $\delta=C\left(\sqrt{2} h_{0}\right)^{s}<\delta_{0}$, in which case $\gamma=\sqrt{1-C^{2}\left(\sqrt{2} h_{0}\right)^{2 s}}>\gamma_{0}$ and $\tau=h_{0}$.

We finally observe that the requirement in (13) that $\tau \leq h_{0}$ places a severe restriction on the mesh norm $h_{X}$ when $L$ is large, given the assumption that $h_{X} \leq$ $\tau / L$. If the theorems are stated not for all $L \geq 1$ but instead for all $L \geq L_{0}$, then it is easily seen that $h_{0}$ in (13) (and hence also in the paragraph that follows it) can be replaced by $L_{0} h_{0}$, since that will be enough to ensure $h_{X} \leq h_{0}$ for $L \geq L_{0}$.

\section{SAmpling TheOREM}

Underlying the $L_{2}$-estimate in Theorem 3.1] is the sampling theorem; see [8, 12]. For completeness, we state it here and show how it can be used to establish easily a weaker version of our main result.

Theorem 4.1. Suppose $X=\left\{x_{1}, \ldots, x_{N}\right\} \subset S^{d}$ is a finite set satisfying $h_{X} \leq$ $1 /(2 L)$. Then, for every $x \in S^{d}$ there exist numbers $a_{j}(x), j=1, \ldots, N$, such that

$$
\sum_{j=1}^{N} a_{j}(x) q\left(x_{j}\right)=q(x), \quad \text { for all } q \in \mathbb{P}_{L}
$$


and such that

$$
\sum_{j=1}^{N}\left|a_{j}(x)\right| \leq 2
$$

Given a fixed $p \in \mathbb{P}_{L}$, let $x_{0} \in S^{d}$ be a point at which $|p(x)|$ takes its maximum value. Then we have

$$
\sum_{j=1}^{N} a_{j}\left(x_{0}\right) p\left(x_{j}\right)=p\left(x_{0}\right)= \pm\|p\|_{L_{\infty}} .
$$

Now choose $w \in V_{X}$ to be

$$
w= \pm \sum_{j=1}^{N} a_{j}\left(x_{0}\right) \Phi\left(\cdot, x_{j}\right)
$$

where the sign is the same as in the preceding equation. Then

$$
(p, w)_{H^{s}}= \pm \sum_{j=1}^{N} a_{j}\left(x_{0}\right)\left(p, \Phi\left(\cdot, x_{j}\right)\right)_{H^{s}}= \pm \sum_{j=1}^{N} a_{j}\left(x_{0}\right) p\left(x_{j}\right)=\|p\|_{L_{\infty}},
$$

so that

$$
\sup _{v \in V_{X} \backslash\{0\}} \frac{(p, v)_{H^{s}}}{\|v\|_{H^{s}}} \geq \frac{(p, w)_{H^{s}}}{\|w\|_{H^{s}}}=\frac{\|p\|_{L_{\infty}}}{\|w\|_{H^{s}}} .
$$

As a special case of the addition theorem for spherical harmonics (cf. 9]), it follows from (2) that

$$
\Phi\left(x_{j}, x_{j}\right):=\sum_{\ell=0}^{\infty} \frac{1}{\left(1+\ell^{2}\right)^{s}} \sum_{k=1}^{N(d, \ell)} Y_{\ell, k}\left(x_{j}\right) Y_{\ell, k}\left(x_{j}\right)=\sum_{\ell=0}^{\infty} \frac{1}{\left(1+\ell^{2}\right)^{s}} \frac{N(d, \ell)}{\omega_{d}}=: \frac{c_{s, d}}{4 \omega_{d}},
$$

where $\omega_{d}$ denotes the surface area of $S^{d}$. Hence, we see from (15) and (14) and the reproducing property of the kernel $\Phi$ that

$$
\|w\|_{H^{s}} \leq \sum_{j=1}^{N}\left|a_{j}\left(x_{0}\right)\right||| \Phi\left(\cdot, x_{j}\right) \|_{H^{s}}=\sum_{j=1}^{N}\left|a_{j}\left(x_{0}\right)\right| \Phi\left(x_{j}, x_{j}\right)^{\frac{1}{2}} \leq \sqrt{c_{s, d} / \omega_{d}} .
$$

Thus from (16) we have

$$
\sup _{v \in V_{X} \backslash\{0\}} \frac{(p, v)_{H^{s}}}{\|v\|_{H^{s}}} \geq \frac{\sqrt{\omega_{d}}}{\sqrt{c_{s, d}}}\|p\|_{L_{\infty}} \geq \frac{1}{\sqrt{c_{s, d}}}\|p\|_{L_{2}} \geq \frac{1}{\sqrt{c_{s, d}}(\sqrt{2} L)^{s}}\|p\|_{H^{s}}
$$

using again in the last step the inverse estimate (11) for spherical polynomials.

This is a weaker result than Theorem 2.1 since the constant now decreases with increasing $L$.

\section{ERROR ANALYSIS FOR THE HYBRID METHOD}

In this section we illustrate the use of the inf-sup condition by using it to give a new error analysis for the hybrid polynomial-plus-radial-basis-function method of Sloan and Sommariva [11. We shall assume in this section that $\Phi=\Phi_{s}$ is the reproducing kernel for the space $H^{s}$ given by (2). In Section 6 we shall show that essentially the same result holds if $\Phi$ is the reproducing kernel of any Hilbert space that is norm equivalent to $H^{s}$. 
To define the hybrid approximation, let $X=X_{N}:=\left\{x_{1}, \ldots, x_{N}\right\} \subset S^{d}$ be a finite point set on $S^{d}$, and let $L \geq 1$ be fixed. Given $f \in H^{s}$, the hybrid approximation approximates $f$ by

$$
f_{X, L}=u_{X, L}+p_{X, L}
$$

where $p_{X, L} \in \mathbb{P}_{L}$ is a polynomial and

$$
u_{X, L}=\sum_{j=1}^{N} \alpha_{j} \Phi\left(\cdot, x_{j}\right)
$$

is a linear combination of radial basis functions centred at the points in $X$, and where the approximation is fixed by the interpolation conditions

$$
f_{X, L}\left(x_{j}\right)=f\left(x_{j}\right), \quad j=1, \ldots, N,
$$

and the side conditions

$$
\sum_{j=1}^{N} \alpha_{j} q\left(x_{j}\right)=0, \quad q \in \mathbb{P}_{L} .
$$

An equivalent formulation, better suited to the present analysis, is: Find $u_{X, L} \in$ $V_{X}$ and $p_{X, L} \in \mathbb{P}_{L}$ such that

$$
\left(u_{X, L}, v_{X}\right)_{H^{s}}+\left(p_{X, L}, v_{X}\right)_{H^{s}}=\left(f, v_{X}\right)_{H^{s}}, \quad v_{X} \in V_{X},
$$

and

$$
\left(q, u_{X, L}\right)_{H^{s}}=0, \quad q \in \mathbb{P}_{L} .
$$

The equivalence follows from (3) and the reproducing property (1) of $\Phi$.

Now assume that $h_{X} \leq \tau / L$, where the parameter $\tau$ is as in Theorems 2.1 . 2.3. That the approximation is well defined can be demonstrated as follows. First, assuming $u_{X, L} \in V_{X}$ and $p_{X, L} \in \mathbb{P}_{L}$ satisfy (18) and (19), we have

$$
\begin{aligned}
\left\|u_{X, L}\right\|_{H^{s}}^{2} & =\left(u_{X, L}, u_{X, L}\right)_{H^{s}} \\
& =\left(f, u_{X, L}\right)_{H^{s}}-\left(p_{X, L}, u_{X, L}\right)_{H^{s}} \\
& =\left(f, u_{X, L}\right)_{H^{s}},
\end{aligned}
$$

where we used (18) and then (19). The Cauchy-Schwarz inequality then gives

$$
\left\|u_{X, L}\right\|_{H^{s}}^{2} \leq\|f\|_{H^{s}}\left\|u_{X, L}\right\|_{H^{s}},
$$

and on cancelling $\left\|u_{X, L}\right\|_{H^{s}}$,

$$
\left\|u_{X, L}\right\|_{H^{s}} \leq\|f\|_{H^{s}} .
$$

The inf-sup condition (4) now gives

$$
\begin{aligned}
\left\|p_{X, L}\right\|_{H^{s}} & \leq \frac{1}{\gamma} \sup _{v_{X} \in V_{X} \backslash\{0\}} \frac{\left(p_{X, L}, v_{X}\right)_{H^{s}}}{\left\|v_{X}\right\|_{H^{s}}} \\
& =\frac{1}{\gamma} \sup _{v_{X} \in V_{X} \backslash\{0\}} \frac{\left(f, v_{X}\right)_{H^{s}}-\left(u_{X, L}, v_{X}\right)_{H^{s}}}{\left\|v_{X}\right\|_{H^{s}}} \\
& \leq \frac{1}{\gamma}\left(\|f\|_{H^{s}}+\left\|u_{X, L}\right\|_{H^{s}}\right) \\
& \leq \frac{2}{\gamma}\|f\|_{H^{s}}
\end{aligned}
$$


where in the last step we used (20). Thus the approximate problem is well posed in $H^{s}$. The fact that the approximate problem has a unique solution then follows from (20) and (21) with $f$ replaced by 0 (since (18) and (19) certainly have a solution when $f=0$ ). In turn, existence of a solution for general $f$ follows from the fact that (18) and (19) together form a square linear system (of dimension $N+N(d, \ell)$ ). Thus the approximation is well defined.

To discuss the error, we first write the exact function $f$ in an analogous way to $f_{X}$ : We write

$$
f=u+p,
$$

where $p \in \mathbb{P}_{L}$ and $u \in H^{s}$ are defined by

$$
(p, q)_{H^{s}}=(f, q)_{H^{s}}, \quad q \in \mathbb{P}_{L},
$$

so that $p$ is the $H^{s}$-orthogonal projection of $f$ on $\mathbb{P}_{L}$, and

$$
u:=f-p .
$$

Clearly, then $u$ and $p$ satisfy equations analogous to (18) and (19),

$$
\begin{aligned}
(u, v)_{H^{s}}+(p, v)_{H^{s}} & =(f, v)_{H^{s}}, \quad v \in H^{s}, \\
(q, u)_{H^{s}} & =0, \quad q \in \mathbb{P}_{L} .
\end{aligned}
$$

On subtracting (18) from (22) (with $v$ replaced by $v_{X} \in V_{X}$ ) and (19) from (23), we obtain

$$
\begin{aligned}
\left(u-u_{X, L}, v_{X}\right)_{H^{s}}+\left(p-p_{X, L}, v_{X}\right)_{H^{s}} & =0, \quad v_{X} \in V_{X} \\
\left(q, u-u_{X, L}\right)_{H^{s}} & =0, \quad q \in \mathbb{P}_{L} .
\end{aligned}
$$

Now define $\tilde{u}_{X} \in V_{X}$ to be the $H^{s}$-orthogonal projection of $u$ on $V_{X}$, that is,

$$
\left(\tilde{u}_{X}, v_{X}\right)_{H^{s}}=\left(u, v_{X}\right)_{H^{s}}, \quad v_{X} \in H^{s}
$$

thus $\tilde{u}_{X}$ is the interpolant of $u$ in the space $V_{X}$ at the points of $X$. (The function $\tilde{u}_{X}$ plays the role of the "elliptic projection" in a finite element analysis. Essentially, it is a convenient function lying in $V_{X}$ that, unlike $u_{X, L}$, differs in a known way from u.) From (24), (25) and (26) we clearly have

$$
\left(\tilde{u}_{X}-u_{X, L}, v_{X}\right)_{H^{s}}+\left(p-p_{X, L}, v_{X}\right)_{H^{s}}=0, \quad v_{X} \in V_{X}
$$

and

$$
\left(q, \tilde{u}_{X}-u_{X, L}\right)_{H^{s}}=\left(q, \tilde{u}_{X}-u\right)_{H^{s}}, \quad q \in \mathbb{P}_{L} .
$$

The inf-sup condition (4) now gives

$$
\begin{aligned}
\left\|p-p_{X, L}\right\|_{H^{s}} & \leq \frac{1}{\gamma} \sup _{v_{X} \in V_{X} \backslash\{0\}} \frac{\left(p-p_{X, L}, v_{X}\right)_{H^{s}}}{\left\|v_{X}\right\|_{H^{s}}} \\
& =-\frac{1}{\gamma} \sup _{v_{X} \in V_{X} \backslash\{0\}} \frac{\left(\tilde{u}_{X}-u_{X, L}, v_{X}\right)_{H^{s}}}{\left\|v_{X}\right\|_{H^{s}}} \\
& \leq \frac{1}{\gamma}\left\|\tilde{u}_{X}-u_{X, L}\right\|_{H^{s}},
\end{aligned}
$$


where we used (27). We also have, using (27) with $v_{X}=\tilde{u}_{X}-u_{X, L}$ and then (28),

$$
\begin{aligned}
\left\|\tilde{u}_{X}-u_{X, L}\right\|_{H^{s}}^{2} & =-\left(p-p_{X, L}, \tilde{u}_{X}-u_{X, L}\right)_{H^{s}} \\
& =-\left(p-p_{X, L}, \tilde{u}_{X}-u\right)_{H^{s}} \\
& \leq\left\|p-p_{X, L}\right\|_{H^{s}}\left\|\tilde{u}_{X}-u\right\|_{H^{s}} \\
& \leq \epsilon\left\|p-p_{X, L}\right\|_{H^{s}}^{2}+\frac{1}{4 \epsilon}\left\|\tilde{u}_{X}-u\right\|_{H^{s}}^{2},
\end{aligned}
$$

where in the last step we used the inequality

$$
a b \leq \epsilon a^{2}+\frac{1}{4 \epsilon} b^{2},
$$

which holds for arbitrary $\epsilon \in(0,1)$. Then from (29) we obtain

$$
\left\|p-p_{X, L}\right\|_{H^{s}}^{2} \leq \frac{\epsilon}{\gamma^{2}}\left\|p-p_{X, L}\right\|_{H^{s}}^{2}+\frac{1}{4 \epsilon \gamma^{2}}\left\|\tilde{u}_{X}-u\right\|_{H^{s}}^{2},
$$

and on choosing $\epsilon=\frac{1}{2} \gamma^{2}$ and simplifying,

$$
\left\|p-p_{X, L}\right\|_{H^{s}}^{2} \leq \frac{1}{\gamma^{4}}\left\|\tilde{u}_{X}-u\right\|_{H^{s}}^{2},
$$

and in turn from (30)

$$
\left\|\tilde{u}_{X}-u_{X, L}\right\|_{H^{s}}^{2} \leq \frac{1}{\gamma^{2}}\left\|\tilde{u}_{X}-u\right\|_{H^{s}}^{2} .
$$

We are now ready to state the main result for the error analysis of the hybrid method.

Theorem 5.1. Let $L \geq 1$ and $X=\left\{x_{1}, \ldots, x_{N}\right\} \subset \mathcal{S}^{d}$ be such that $h_{X} \leq \tau / L$, where $\tau$ is as in Theorem 2.1, and let $\Phi=\Phi_{s}$ be the reproducing kernel in the space $H^{s}$ for some fixed $s>d / 2$. Moreover, given $f \in H^{s}$ let $f_{X, L}=u_{X, L}+p_{X, L}$, where $u_{X, L} \in V_{X}$ and $p_{X, L} \in \mathbb{P}_{L}$ are the solutions of (18) and (19). Then,

$$
\left\|f-f_{X, L}\right\|_{L^{2}} \leq c h^{s} \inf _{q \in \mathbb{P}_{L}}\|f-q\|_{H^{s}} \leq C h^{s}\|f\|_{H^{s}},
$$

and, if $f \in H^{2 s}$,

$$
\left\|f-f_{X, L}\right\|_{L^{2}} \leq c h^{2 s} \inf _{q \in \mathbb{P}_{L}}\|f-q\|_{H^{2 s}} \leq C h^{2 s}\|f\|_{H^{2 s}},
$$

with $C$ independent of $X$ and $L$.

Here, and in the following work, $C$ denotes a generic constant, whose value at each occurrence may be different.

Proof. The first step is to note from Theorem 3.1 that

$$
\begin{aligned}
\left\|f-f_{X, L}\right\|_{L^{2}} & \leq C h^{s}\left\|f-f_{X, L}\right\|_{H^{s}} \\
& \leq C h^{s}\left(\left\|u-u_{X, L}\right\|_{H^{s}}+\left\|p-p_{X, L}\right\|_{H^{s}}\right),
\end{aligned}
$$

where we used the fact that $f-f_{X, L}$ vanishes at the points of $X$, together with $f=u+p$ and $f_{X, L}=u_{X, L}+p_{X, L}$. Next we note that (31) and (32) give

$$
\left\|p-p_{X, L}\right\|_{H^{s}} \leq C\left\|\tilde{u}_{X}-u\right\|_{H^{s}}
$$

and

$$
\left\|u-u_{X, L}\right\|_{H^{s}} \leq\left\|u-\tilde{u}_{X}\right\|_{H^{s}}+\left\|\tilde{u}_{X}-u_{X, L}\right\|_{H^{s}} \leq C\left\|\tilde{u}_{X}-u\right\|_{H^{s}}
$$


and putting these together,

$$
\left\|f-f_{X, L}\right\|_{L^{2}} \leq C h^{s}\left\|\tilde{u}_{X}-u\right\|_{H^{s}} .
$$

Since $\tilde{u}_{X}$ is the interpolant of $u$ on $V_{X}$, it follows from Corollary 3.3 that

$$
\left\|\tilde{u}_{X}-u\right\|_{H^{s}} \leq C h_{X}^{s}\|u\|_{H^{2 s}}
$$

and also we have that

$$
\left\|\tilde{u}_{X}-u\right\|_{H^{s}} \leq\|u\|_{H^{s}}
$$

since $\tilde{u}_{X}$ is the $H^{s}$-orthogonal projection of $u$ on $V_{X}$. It therefore follows from (33) that

$$
\left\|f-f_{X, L}\right\|_{L^{2}} \leq C h^{t}\|u\|_{H^{t}} \quad \text { for } t=s, 2 s .
$$

Finally, we may note that $p$ has the Fourier series representation

$$
p=\sum_{\ell=0}^{L} \sum_{k=1}^{N(d, \ell)} \hat{f}_{\ell, k} Y_{\ell, k}
$$

which is the $H^{t}$-orthogonal projection of $f$ on $\mathbb{P}_{L}$ for all $t \geq 0$. It follows that $p$ and $u=f-p$ are orthogonal in $H^{t}$ for all $t \geq 0$, and hence that

$$
\|u\|_{H^{t}}=\|f-p\|_{H^{t}}=\inf _{q \in \mathbb{P}_{L}}\|f-q\|_{H^{t}} \leq\|f\|_{H^{t}}
$$

The theorem now follows from (35) and (36).

\section{The native space Corresponding to $\Phi$}

In this section we broaden the assumptions on $\Phi$, by replacing (2) with the more general assumption

$$
\Phi(x, y)=\sum_{\ell=0}^{\infty} \sum_{k=1}^{N(d, \ell)} a_{\ell} Y_{\ell, k}(x) Y_{\ell, k}(y)
$$

with

$$
a_{\ell} \sim\left(1+\ell^{2}\right)^{-s}
$$

for some $s>d / 2$, where (38) means that there exist positive constants $c_{1}$ and $c_{2}$, independent of $\ell$, such that

$$
c_{1}\left(1+\ell^{2}\right)^{-s} \leq a_{\ell} \leq c_{2}\left(1+\ell^{2}\right)^{-s}, \quad \ell \in \mathbb{N} .
$$

In this situation the "native space" $N_{\Phi}$ that corresponds to the kernel $\Phi$ is the Hilbert space

$$
N_{\Phi}:=\left\{u \in L_{2}\left(S^{d}\right): \sum_{\ell=0}^{\infty} \sum_{k=1}^{N(d, \ell)} \frac{\left|\widehat{u}_{\ell, k}\right|^{2}}{a_{\ell}}<\infty\right\},
$$

and the norm in $N_{\Phi}$ is defined by the inner product

$$
(u, v)_{N_{\Phi}}:=\sum_{\ell=0}^{\infty} \sum_{k=1}^{N(d, \ell)} \frac{1}{a_{\ell}} \widehat{u}_{\ell, k} \widehat{v}_{\ell, k} .
$$

The space $N_{\Phi}$ is a reproducing kernel Hilbert space with reproducing kernel $\Phi(x, y)$. The norms in $N_{\Phi}$ and $H^{s}$ are clearly equivalent. 
Our purpose in this section is to establish that the principal conclusions of this paper are unaltered if throughout we replace $H^{s}$ by $N_{\Phi}$. First, Theorem 2.1 is replaced by:

Theorem 6.1. Let $\Phi$ be a kernel of the form (37) satisfying (39) for some $s>d / 2$. Then there exist constants $\gamma^{\prime}>0$ and $\tau^{\prime}>0$ depending only on $d$ and $s$ such that for all $L \geq 1$ and all $X=\left\{x_{1}, \ldots, x_{N}\right\} \subset S^{d}$ satisfying $h_{X} \leq \tau^{\prime} / L$ the following inequality holds:

$$
\sup _{v \in V_{X} \backslash\{0\}} \frac{(p, v)_{N_{\Phi}}}{\|v\|_{N_{\Phi}}} \geq \gamma^{\prime}\|p\|_{N_{\Phi}}, \quad p \in \mathbb{P}_{L} .
$$

Proof. Note first that for $f \in C\left(S^{d}\right)$ the interpolant $I_{X} f$ is now the $N_{\Phi^{-} \text {-orthogonal }}$ projection of $f$ on $V_{X}$, since

$$
0=I_{X} f\left(x_{j}\right)-f\left(x_{j}\right)=\left(I_{X} f-f, \Phi\left(\cdot, x_{j}\right)\right)_{N_{\Phi}}, j=1, \ldots, N .
$$

Thus, Proposition 2.4 gives

$$
\begin{aligned}
\sup _{v \in V_{X} \backslash\{0\}} \frac{(p, v)_{N_{\Phi}}}{\|v\|_{N_{\Phi}}} & =\left\|I_{X} p\right\|_{N_{\Phi}} \\
& \geq c_{2}^{-1 / 2}\left\|I_{X} p\right\|_{H^{s}} \\
& \geq c_{2}^{-1 / 2}\left(1-\delta^{2}\right)\|p\|_{H^{s}},
\end{aligned}
$$

where in the last step we used (12). Now the argument proceeds as in the proof of Theorem 2.3, with $\gamma^{\prime}=\gamma / \sqrt{c_{2}}$.

The hybrid approximation now takes the same form as (17), but instead of (18) and (19) we have

$$
\begin{aligned}
\left(u_{X, L}, v_{X}\right)_{N_{\Phi}}+\left(p_{X, L}, v_{X}\right)_{N_{\Phi}} & =\left(f, v_{X}\right)_{N_{\Phi}}, & v_{X} \in V_{X}, \\
\left(q, u_{X, L}\right)_{N_{\Phi}} & =0, & q \in \mathbb{P}_{L} .
\end{aligned}
$$

Instead of Theorem 5.1 we obtain:

Theorem 6.2. Let $\Phi$ be a kernel of the form (37) satisfying (39) for some $s>d / 2$. Moreover, let $L \geq 1$ and $X=\left\{x_{1}, \ldots, x_{N}\right\} \subset S^{2}$ be such that $h_{X} \leq \tau^{\prime} / L$ where $\tau^{\prime}$ is as in Theorem 6.1. Given $f \in N_{\Phi}$, let $f_{X, L}=u_{X, L}+p_{X, L}$, where $u_{X, L} \in V_{X}$ and $p_{X, L} \in \mathbb{P}_{L}$ are solutions of (40) and (41). Then,

$$
\left\|f-f_{X, L}\right\|_{L^{2}} \leq C^{\prime} h^{s}\|f\|_{H^{s}}
$$

and, if $f \in H^{2 s}$,

$$
\left\|f-f_{X, L}\right\|_{L^{2}} \leq C^{\prime} h^{2 s}\|f\|_{H^{2 s}},
$$

with $C^{\prime}$ independent of $X$ and $L$.

The proof is exactly the same as for the proof of Theorem 5.1 if we replace $H^{s}$ by $N_{\Phi}$ throughout, if we note that the modified version of (34) still holds because of the norm equivalence of $H^{s}$ and $N_{\Phi}$.

\section{ACKNOWLEDGEMENTS}

The support of the Australian Research Council under its Centres of Excellence program is gratefully acknowledged. The authors are also indebted to Dr. Andy Wathen for insight into the inf-sup condition. 


\section{REFERENCES}

[1] I. Babuska, The finite element method with Lagrangian multipliers, Numer. Math., 20 (1973), pp. 179-192. MR0359352 (50:11806)

[2] F. Brezzi, On the existence, uniqueness and approximation of saddle-point problems arising from Lagrangian multipliers, Rev. Française Automat. Informat. Rech. Opér. Anal. Numer., 8 (1974), pp. 129-151. MR0365287 (51:1540)

[3] J. Duchon, Sur l'erreur d'interpolation des fonctions de plusieurs variables par les $D^{m}$ splines, Rev. Française Automat. Informat. Rech. Opér. Anal. Numer., 12 (1978), pp. 325334. MR.519016 (80j:41052)

[4] H.C. Elman, D.J. Silvester and A.J. Wathen, Finite Elements and Fast Iterative Solvers: with Applications in Incompressible Fluid Dynamics, Oxford University Press, New York, 2005. MR2155549 (2006f:65002)

[5] Q. Le Gia, F. Narcowich, J. Ward and H. Wendland, Continuous and discrete least-squares approximation by basis functions on spheres, J. Approx. Theory, 143 (2006), pp. 124-133. MR2271729 (2007k:41078)

[6] S. Hubbert and T.M. Morton, A Duchon framework for the sphere, J. Approx. Theory, 129 (2004), pp. 28-57. MR2070179 (2005h:41047)

[7] S. Hubbert and T.M. Morton, $L_{p^{-}}$error estimates for radial basis function interpolation on the sphere, J. Approx. Theory, 129 (2004), pp. 58-77. MR2070180 (2005d:41005)

[8] K. Jetter, J. Stöckler and J. Ward, Error estimates for scattered data interpolation on spheres, Math. Comput., 68 (1999), pp. 733-747. MR1642746 (99i:41032)

[9] C. Müller, Spherical Harmonics, Springer-Verlag, Berlin, 1966. MR0199449 (33:7593)

[10] F.J. Narcowich, J.D. Ward and H. Wendland, Sobolev bounds on functions with scattered zeros, with applications to radial basis functions surface fitting, Maths. Comput., 74 (2005), pp. 743-763. MR.2114646(2005k:41051)

[11] I.H. Sloan and A. Sommariva, Approximation on the sphere using radial basis functions plus polynomials, Advances in Computational Mathematics, 29 (2008), pp. 147-177. MR2420870

[12] H. Wendland, Scattered Data Approximation, Cambridge Monographs on Applied and Computational Mathematics, Cambridge University Press, Cambridge, UK, 2005. MR 2131724 (2006i:41002)

School of Mathematics and Statistics, University of New South Wales, Sydney, NSW, 2052, Australia

E-mail address: i.sloan@unsw.edu.au

Department of Mathematics, University of Sussex, Brighton, BN1 9RF, England

E-mail address: h.wendland@sussex.ac.uk 